Notfall Rettungsmed 2014 - 17:286-286

DOI 10.1007/s10049-013-1780-0

Online publiziert: 14. Mai 2014

(c) Springer-Verlag Berlin Heidelberg 2014

\section{U. Zeymer}

Klinikum Ludwigshafen und Institut für Herzinfarktforschung, Ludwigshafen
Die Anzahl der wegen Herzinsuffizienz in deutschen Krankenhäusern behandelten Patienten ist seit Jahren ständig zunehmend. Dies ist unter anderem durch die ansteigende Anzahl älterer Menschen bedingt. Darüber hinaus überleben mehr Patienten einen akuten Herzinfarkt und können im weiteren Verlauf durch die ischämisch bedingte Einschränkung der linksventrikulären Funktion eine Herzinsuffizienz entwickeln. Im Jahr 2011 war die Herzinsuffizienz (ICD I50) die zweithäufigste Einzeldiagnose bei vollstationär behandelten Patienten, und die Fallzahl betrug 380.000. Insgesamt war bei über 465.000 Patienten die Ursache der stationären Aufnahme eine Herzinsuffizienz.

Die Anzahl der an Herzinsuffizienz Verstorbenen pro 100.000 Einwohner betrug $56 \mathrm{im}$ Jahr 2011, und insgesamt verstarben in Deutschland über 45.000 Menschen an dieser Erkrankung. Die Prognose der Patienten mit Herzinsuffizienz nach erstmaliger akuter Dekompensation ist schlechter als die der meisten Krebserkrankungen. Diese Fakten und Zahlen belegen die große medizinische und ökonomische Bedeutung der Erkrankung. Daher widmet sich dieses Schwerpunktthema in Notfall + Rettungsmedizin der Herzinsuffizienz.

\section{》) Luftnot ist eines der Leitsymptome der Herzinsuffizienz}

Dyspnoe ist eines der Leitsymptome der Herzinsuffizienz, aber Luftnot kann auch durch eine Reihe weiterer Erkrankungen verursacht werden. Insbesondere in der präklinischen Phase ist die differenzialdiagnostische Einordnung des Symptoms Luftnot häufig schwierig. Andererseits ist die möglichst schnelle Einlei- tung einer Therapie in vielen Fällen notwendig, um eine klinische Verschlechterung des Patienten zu verhindern. Einer der häufigsten Gründe für eine Krankenhauseinweisung bei Herzinsuffizienz ist ein Therapiefehler, z. B. durch eine zu große Trinkmenge oder das Weglassen von Medikamenten. Eine Medikamentenanamnese ist daher neben der körperlichen Untersuchung und Auskultation eine der wichtigsten diagnostischen Maßnahmen in der Akutsituation.

Bei der Differenzialdiagnostik im Krankenhaus haben die Echokardiographie und die Bestimmung der Biomarker die größte Bedeutung, und diese Maßnahmen sollten in jeder Notaufnahme verfügbar sein. Die Grundpfeiler der Therapie stellen weiterhin die Gabe von Diuretika und Nitraten dar, während die Inotropika und Vasopressoren nur sehr zurückhaltend eingesetzt werden sollten. Dabei ist bemerkenswert, dass sich die medikamentöse Therapie in den letzten Jahren wenig geändert hat und wirkliche innovative Therapieoptionen wie z. B. Serelaxin möglicherweise erst in den nächsten Jahren zu erwarten sind.

Ein neuer Ansatz in der Akut- und langfristigen Versorgung der Patienten mit Herzinsuffizienz ist die Einrichtung von speziell für diese Patienten geschaffenen Herzinsuffizienzeinheiten. Hier kann eine sorgfältige Indikationsstellung für über die medikamentöse Therapie hinausgehende Maßnahmen getroffen werden.

Auch wegen der leider kontinuierlich zurückgehenden Anzahl von Herztransplantationen in Deutschland nimmt die Bedeutung der mechanischen Kreislaufunterstützung zu. Die häufigste Anwendung dieser Systeme findet in de Akutphase, z. B. bei Patienten mit infarktbedingtem kardiogenem Schock, statt. Während der Stellenwert der intraaortalen Ballonpumpe (IABP) nach den Ergebnissen der IABP-Shock-II-Studie zurückgegangen ist, nimmt der Einsatz invasiverer Systeme wie der Impella und der perkutanen Herzlungenmaschinen zu. Hier sind allerdings prospektive Studien zu fordern, um den klinischen Wert dieser Systeme besser definieren zu können.

Die mechanische Unterstützung als Dauertherapie und die Unterstützung bis zur Erholung des Herzens werden zunehmend häufiger eingesetzt. Diese Patienten können in vielen Fällen mit mechanischer Kreislaufunterstützung nach Hause entlassen werden und müssen bei Problemen und Komplikationen vom Notarzt erstversorgt werden. Somit sind Kenntnisse über Wirkungsweise und Komplikationsmöglichkeiten dieser Systeme von allen in der Akutmedizin tätigen Medizinern notwendig. Die Herzinsuffizienz ist auf dem Weg zur Volkskrankheit und die Notarzteinsätze deswegen werden zunehmen. Daher sind gute Kenntnisse über Differenzialdiagnostik und Therapie für den Akutmediziner von großer Bedeutung.

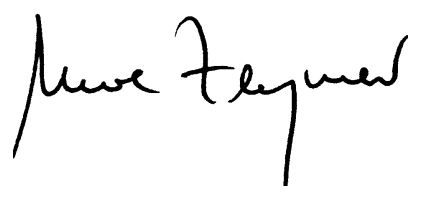

Prof. Dr. Uwe Zeymer



Einhaltung ethischer Richtlinien

Interessenkonflikt. U. Zeymer gibt an, dass kein Interessenkonflikt besteht. 